\title{
Seamless Advective Blending of Total Precipitable Water Retrievals from Polar-Orbiting Satellites*
}

\author{
ANTHONY J. WimMERS AND CHRISTOPHER S. VELDEN \\ Cooperative Institute for Meteorological Satellite Studies, University of Wisconsin-Madison, Madison, Wisconsin
}

(Manuscript received 22 June 2010, in final form 22 October 2010)

\begin{abstract}
Conventional methods of viewing and combining retrieved geophysical fields from polar-orbiting satellites often complicate the work of end users because of the erratic time differences between overpasses, the significant time gaps between elements of a composite image, or simply the different requirements for interpretation between contributing instruments. However, it is possible to mitigate these issues for any number of retrieved quantities in which the tracer lifetime exceeds the sampling time. This paper presents a method that uses "advective blending" to create high-fidelity composites of data from polar-orbiting satellites at high temporal resolution, including a characterization of error as a function of time gap between satellite overpasses. The method is especially effective for tracers with lifetimes of longer than $7 \mathrm{~h}$. Examples are presented using microwave-based retrievals of total precipitable water (TPW) over the ocean, from the Cooperative Institute for Meteorological Satellite Studies (CIMSS) Morphed Integrated Microwave Imagery at CIMSS TPW product (MIMIC-TPW). The mean average error of a global $0.25^{\circ} \times 0.25^{\circ}$ product at 1 - $\mathrm{h}$ resolution is $0.5-2 \mathrm{~mm}$, which is very reasonable for most applications.
\end{abstract}

\section{Introduction}

Imagery of atmospheric variables from polar-orbiting meteorological satellites is often underutilized when the only available presentation is with the original singlepass data. Composites of sequential passes are possible every $12 \mathrm{~h}$ for most polar orbiters, but the time difference and data gaps between overpass swaths often render these composites inferior to alternatives from geostationary satellite imagery and make them difficult for an analyst to use. Composites between multiple polar-orbiting satellites with similar sensors can improve the temporal consistency of the product. However, other issues can emerge, such as erratic time differences between the various components and artifacts from insufficient satellite intercalibration. If the final image product does not fit the user's implicit or explicit requirements for ease of use and fidelity to the observed system, then it will

\footnotetext{
* Supplemental information related to this paper is available at the Journals Online Web site.

Corresponding author address: Anthony J. Wimmers, CIMSS, 1225 W. Dayton St., Madison, WI 53706.

E-mail:wimmers@ssec.wisc.edu
}

probably be neglected in favor of other, possibly more limited, products.

Therefore, novel approaches to visualization are often necessary to create products that improve on the presentation of the original data alone. The tools described in this paper demonstrate that often this issue with polarorbiting satellite data is merely a problem of presentation rather than an intrinsic liability. Here, we present a method of advective blending that combines data from several polar-orbiting satellites and produces an effectively seamless and high temporal resolution final product. The demonstrative variable in this case is total precipitable water (TPW) derived from microwave frequencies. However, alternative variables exhibiting quasi-conservative qualities derived from other sensor frequencies could also be considered for this approach.

The algorithm developed for this application is the Cooperative Institute for Meteorological Satellite Studies (CIMSS) Morphed Integrated Microwave Imagery at CIMSS TPW product (MIMIC-TPW). Microwavederived TPW is especially well suited for this approach because of three particular features. First, it is available from multiple satellite platforms at a similar resolution and with very little intercalibration error. Second, it is derived continuously over water, so it can be processed 
TABLE 1. Satellite sensors contributing to the TPW retrieval.

\begin{tabular}{llccccc}
\hline \hline Satellite & Sensor & $\begin{array}{c}\text { Channels (GHz) } \\
\text { (vertical polarization) }\end{array}$ & $\begin{array}{c}\text { Instantaneous FOV* } \\
(\mathrm{km} \text {; for the “footprint”) }\end{array}$ & $\begin{array}{c}\text { Swath } \\
\text { width (km) }\end{array}$ & $\begin{array}{c}\text { Equator crossing } \\
\text { time (UTC) } \\
\text { (7-22 Jul 2009) }\end{array}$ & Reference \\
\hline$F-13$ & SSM/I & $19.3,22.2,37.0$ & $43 \times 69,40 \times 60,28 \times 37$ & 1400 & 1822 & Raytheon (2000) \\
$F-16, F-17$ & SSMI/S & $19.3,22.2,37.0$ & $73 \times 47,73 \times 47,41 \times 31$ & 1707 & 1927,1728 & NOAA/OSDPD (2002) \\
Aqua & AMSR-E & $18.9,23.8,36.5$ & $27 \times 16,31 \times 18,14 \times 8$ & 1445 & 1330 & NASA MSFC (2001) \\
\hline
\end{tabular}

* FOV: field of view for the three sensor channels, respectively.

without gaps due to clouds. Third, it can be treated as a quasi-conservative tracer over the time scale of a typical gap between separate satellite passes (1-14 h), meaning that it will comply in most cases with the algorithm's advection treatment. An improved TPW product has many potential benefits for oceanic and coastal forecasting, including the monitoring of tropical waves and cyclones (R. Berg, National Hurricane Center Tropical Analysis and Forecast Branch, 2008, personal communication), aerosol layers (Dunion 2011), and "atmospheric rivers" (Newell et al. 1992).

Kidder and Jones (2007) demonstrate a novel multiplatform compositing method for TPW from the Special Sensor Microwave Imager (SSM/I) and the Advanced Microwave Sounding Unit (AMSU) that focuses on nearreal-time intercalibration and more recently includes other data sources including GPS retrievals over North America. While this method does perform some blending, the product retains the data in the positions of the observations without advection. Previous efforts at advective blending focus primarily on the short-term synthesis and extrapolation of precipitation retrievals from radar (Hohti et al. 2000; Germann and Zawadzki 2002) and from polarorbiting satellites (Grose et al. 2002; Joyce et al. 2004). Wimmers and Velden (2007) describe a synthesis of passive microwave observations specialized for tropical cyclones. However, a similar application to longer-lived atmospheric tracers has not been studied or applied. This might be because of a supposed reluctance of forecasters and analysts to use "synthetic" retrieval fields whose accuracy is not characterized as well as the original observations. We will attempt to overcome this concern with a thorough characterization and quantification of the error in the MIMICTPW technique.

This paper briefly discusses the satellite data used in the MIMIC-TPW product (section 2), describes the advective blending algorithm (section 3 ), characterizes the frequency of satellite input for this application (section 4), presents a product validation (section 5), illustrates examples of the product's potential for forecasting and nowcasting applications (section 6), and discusses the broader implications of this approach to representing polar-orbiting satellite data (section 7). The MIMIC-TPW product will be discussed as a retrospective diagnostic composite product (emphasizing interpolation) and as a real-time operational analytic/prognostic product (emphasizing extrapolation).

\section{Data sources}

\section{a. Satellite sensors and TPW calculation}

The approach outlined here places a higher than normal priority on accurate satellite intercalibration because the benefits of seamless image transitions cannot be fully realized without it. The satellite products also need to have a similar spatial resolution; otherwise, the resolution artifacts would be too obvious in the final product. Therefore, the best satellite-based microwave sensors for an advected TPW composite are the SSM/I on board the Defense Meteorological Satellite Program's (DMSP) F-13 satellite (available during the test period but no longer available at the time of writing), the $F-16$ Special Sensor Microwave Imager Sounder (SSMI/S), the $F-17$ SSMI/S, and the National Aeronautics and Space Administration's (NASA) Advanced Microwave Scanning Radiometer-Earth Observing System (EOS) (AMSR-E) on board the Aqua satellite (Table 1). The TPW products of the Tropical Rainfall Measuring Mission Microwave Imager, WindSat, and Coriolis are not used, because their retrieval methods are different enough from those of SSM/I, SSMI/S, and AMSR-E to leave small but significant intercalibration artifacts.

The TPW product is generated as a linear function of the 19-, 22-, and 37-GHz satellite sensors, as in Alishouse et al. (1990), and does not require a first guess from an atmospheric model. Fortuitously, the $F-13, F-16$, and $F-17$ satellites have the same versions of sensor instruments operating at these frequencies, so TPW retrievals from these platforms can be used interchangeably without any extra intercalibration. (We find in section 5 that the TPW retrievals between these three platforms are within less than $1 \mathrm{~mm}$.) The same instrument behavior is expected with subsequent SSMI/S sensors to be launched. The TPW values derived from AMSR-E have a small bias with respect to the other sensors, and so a histogram-matching 
TABLE 2. Advecting wind profile weighting functions organized by latitude. Weights between $25^{\circ}$ and $50^{\circ}$ latitude are calculated by linear interpolation.

\begin{tabular}{ccc}
\hline \hline Height $(\mathrm{hPa})$ & Weight, $\leq 25^{\circ} \mathrm{N} / \mathrm{S}$ & Weight, $\geq 50^{\circ} \mathrm{N} / \mathrm{S}$ \\
\hline 700 & 0.160 & 0.138 \\
850 & 0.280 & 0.345 \\
1000 & 0.560 & 0.517 \\
\hline
\end{tabular}

correction is applied to these values. It is sufficient for our purposes to update this intercalibration about once a year to account for sensor drift.

\section{b. Ancillary numerical model winds}

The data advection process uses contemporaneous global model-derived winds near the surface. We employ the Global Forecast System's (GFS) global analysis and 6-h resolution forecasts (Kalnay and Baker 1990) available from the National Oceanic and Atmospheric Administration's (NOAA) NOAAPORT data distribution network, and interpolate to every hour. The bulk of the TPW resides in the lower troposphere; therefore, the model multilevel wind fields are condensed into a twodimensional advection field by calculating a weighted average of the 1000-, 850-, and 700-hPa wind fields (Table 2). The weights vary a small amount with latitude. This weighting function was determined empirically by trial and error, but it approximates the climatological average distribution of specific humidity in the lower troposphere as a function of latitude (Piexoto and Oort 1992).

\section{Algorithm}

\section{a. Swath advection}

The term "swath" is used here to describe the data from a single ascending or descending pass of a polar satellite. Each microwave-derived TPW swath is assumed to be accurate for a number of hours before and after the retrieval time. To represent the TPW swath at times several hours away from the retrieval time, we treat TPW as a purely conservative tracer advected by a background wind field (section 2). Obviously, this presumed evolution of TPW is an approximation to the actual evolution of the TPW field because in our scheme it advects as a simple two-dimensional field and undergoes no phase change of water. However, the product validation in section 4 shows that this approximation is very effective over time spans of several hours. Figure 1 shows this advection process at work. The distorted swath boundaries in each image indicate the extent of the total advection. This process creates "synthetic" data fields at any desired temporal spacing and time length to be used in the subsequent stage of the algorithm. Each point in the original retrieval receives its own trajectory over a given time span, and the synthetic data corresponding to an advected swath are generated by interpolation of these trajectory points at a moment in time onto a regular grid. This method prevents the propagation of error due to repeated regridding.

\section{b. Compositing}

The method for producing composite products is a conventional time-weighted interpolation. The principal difference between this method and most other forms of time-weighted averaging is that the inputs are already advected to the valid time, which creates a much smoother end product.

The first step in compositing is to automatically sift through the abundance of synthetic data available at the valid time and choose the parts of the swaths whose original observations occur closest to the valid time. This minimizes the advection time of the input data (Fig. 2a). Compositing is done separately for data before and after the valid time to produce two composites: one of data "earlier" than the valid time and one of data "later" than the valid time (Fig. 2b).

Next, the temporal weights are calculated. The weights are calculated as a simple time-weighted average:

$$
\begin{aligned}
& w_{\text {earlier }}=\Delta t_{\text {later }} /\left(\Delta t_{\text {earlier }}+\Delta t_{\text {later }}\right) \text { and } \\
& w_{\text {later }}=1-w_{\text {earlier }},
\end{aligned}
$$

where

$$
\begin{aligned}
\Delta t_{\text {earlier }} & =t_{\text {valid }}-t_{\text {earlier }} \text { and } \\
\Delta t_{\text {later }} & =t_{\text {later }}-t_{\text {valid }},
\end{aligned}
$$

and where each of the variables is two-dimensional by latitude and longitude (Figs. 2c and 2d). The final product is the weighted average of the earlier and later composites (Fig. 3) in much the same manner as Joyce et al.'s (2004) morphed precipitation fields.

Although this process is demonstrated with an example from a single time period, the benefits of this method become more apparent when the product is in motion (section 6 ). As the product progresses with time, each contributing swath is gradually introduced and expired without a discontinuity, and the result is an impressively smooth progression of images in spite of the apparent chaos of the weighting functions in Fig. $2 d$.

\section{c. Data extrapolation at coasts}

The microwave TPW retrieval is valid only over water because of the high sensitivity of the contributing microwave channels to surface emissivity. Therefore, landmasses are masked from the MIMIC-TPW product and 
a) Advection back to 0300 UTC (-6.37 hr)

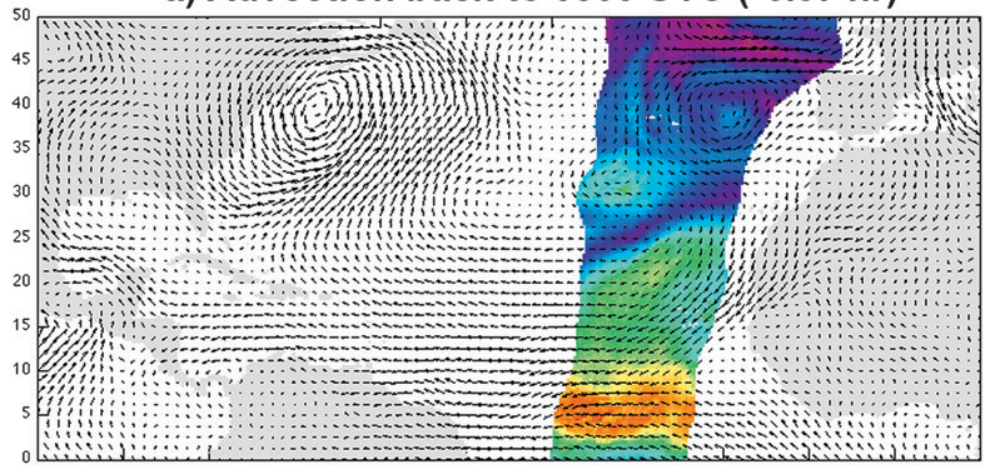

b) Original swath: "0922 UTC" (0915-0929 UTC)

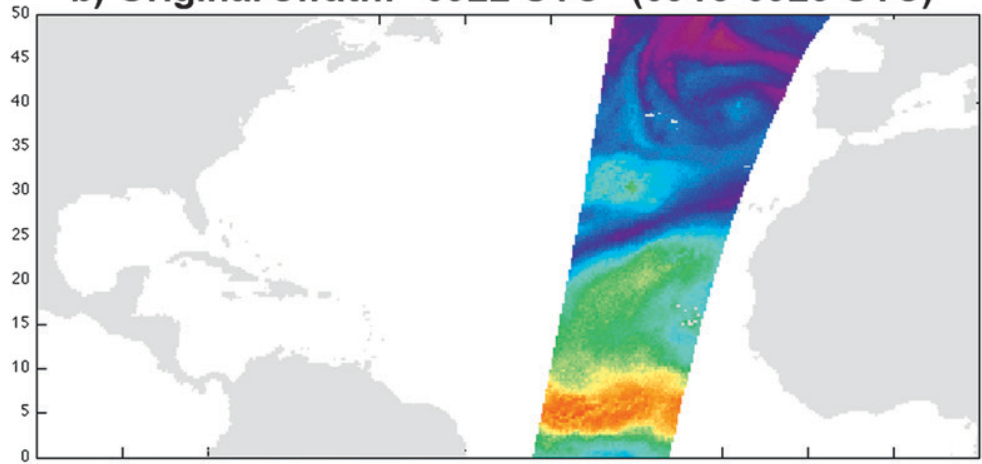

c) Advection forward to 1500 UTC (+5.63 hr)

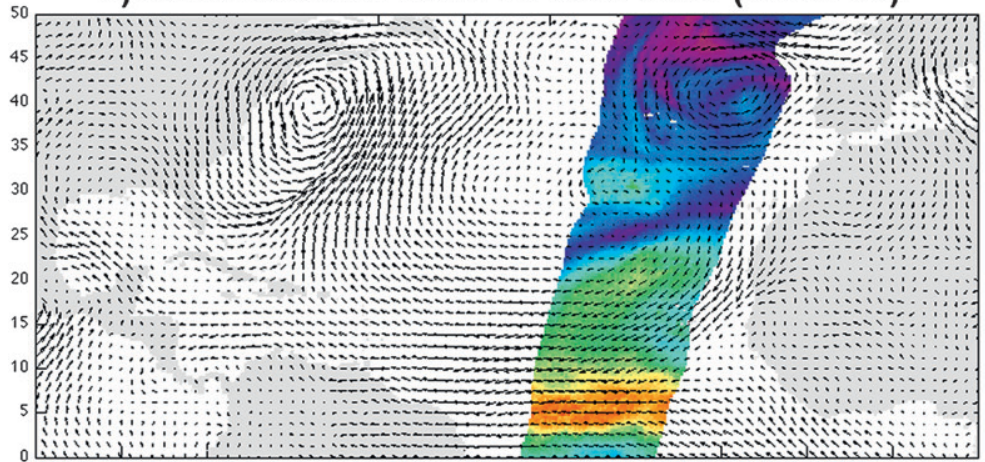

d) Advection forward to 2100 UTC (+11.63 hr)

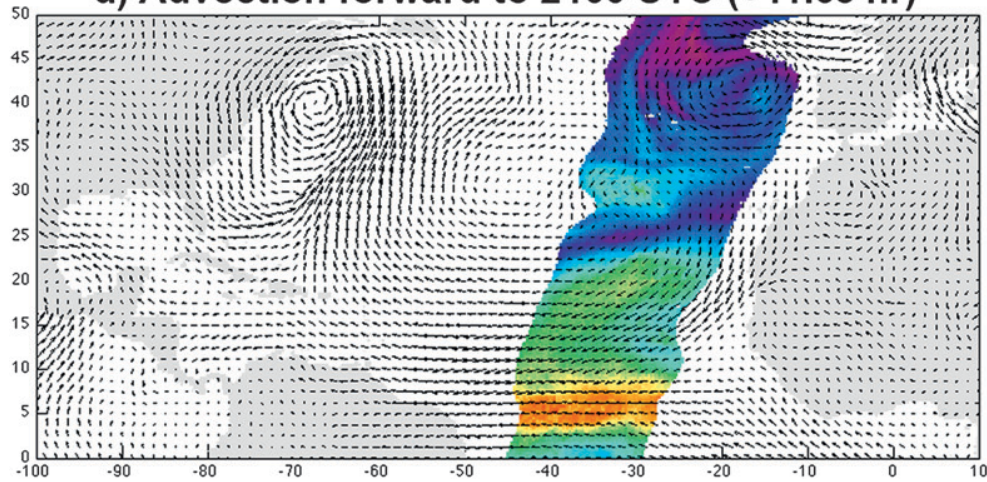

FIG. 1. The advection process for a single TPW swath from the $F-16 \mathrm{SSMI} / \mathrm{S}$ at approximately 0922 UTC (0915-0929 UTC) 22 Jun 2009. The color scale is as in Figs. 3 and 4. 


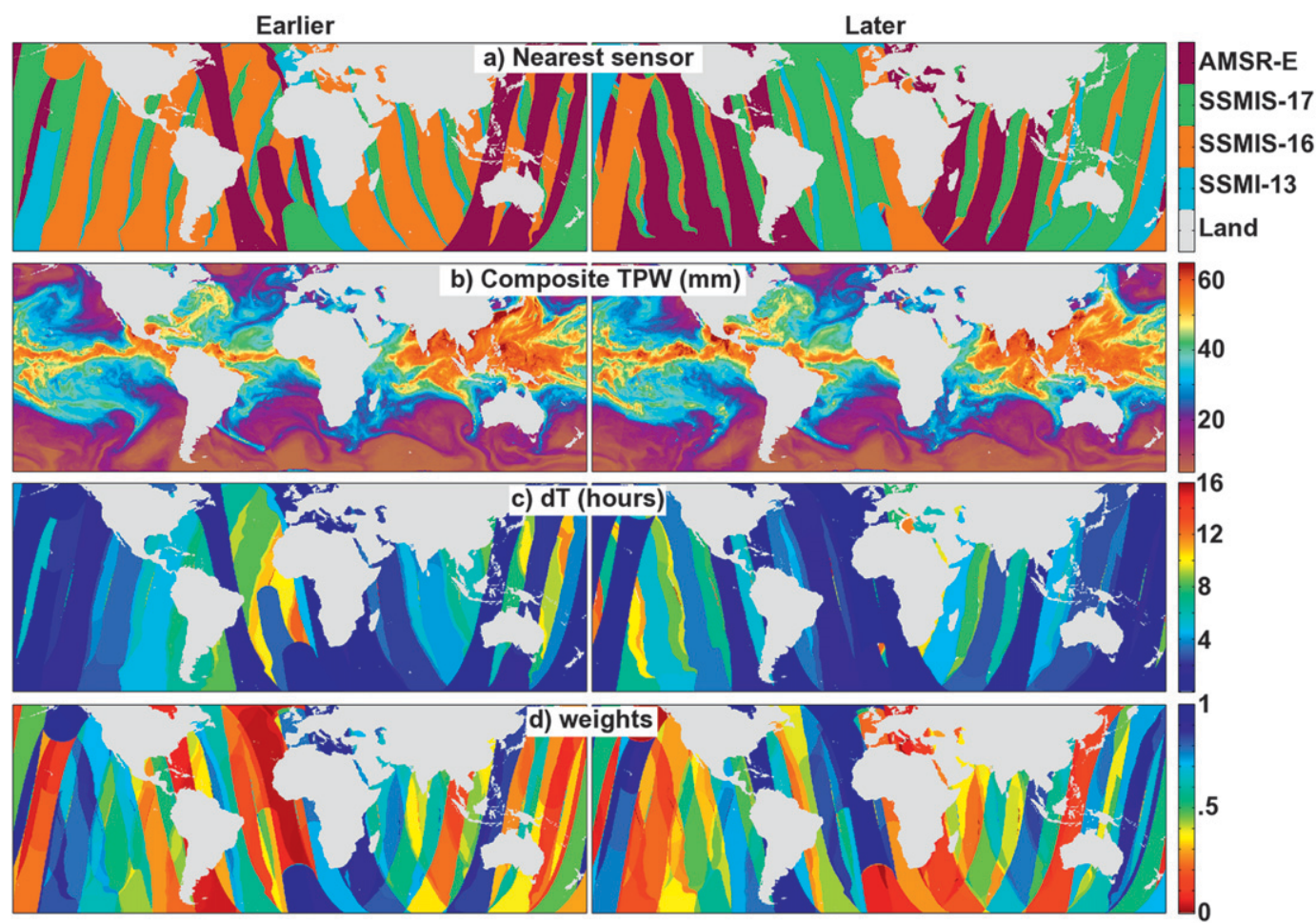

FIG. 2. Components of the MIMIC-TPW compositing process at 1800 UTC 22 Jun 2009. (Final product shown in Fig. 3.)

the domain is limited to $60^{\circ} \mathrm{N}-60^{\circ} \mathrm{S}$ in order to avoid polar ice. Likewise, any single retrieval can be corrupted by a partial contribution of land, so the region of valid data is an area that recedes slightly oceanward from the coasts. Clearly, the final product would have very distracting artifacts at the coasts if this data-void buffer region around the coasts was not corrected with some form of extrapolated data values.

We have found that the most effective method for filling in TPW values in this missing-data region is to apply the "inpainting" technique of Bertalmío et al. (2001). The technique works by filling data-void regions of an image with the steady-state solution to the Navier-Stokes equation, where image intensity substitutes for the equation's streamfunction. The result is a filled region with sharper and more continuous gradients than is produced by ordinary interpolation. Figure 4 shows that this approach works well with the general patterns of a TPW distribution.

\section{d. Real-time operation}

Naturally, a real-time or near-real-time composite product lacks the later (future) data necessary for a temporal

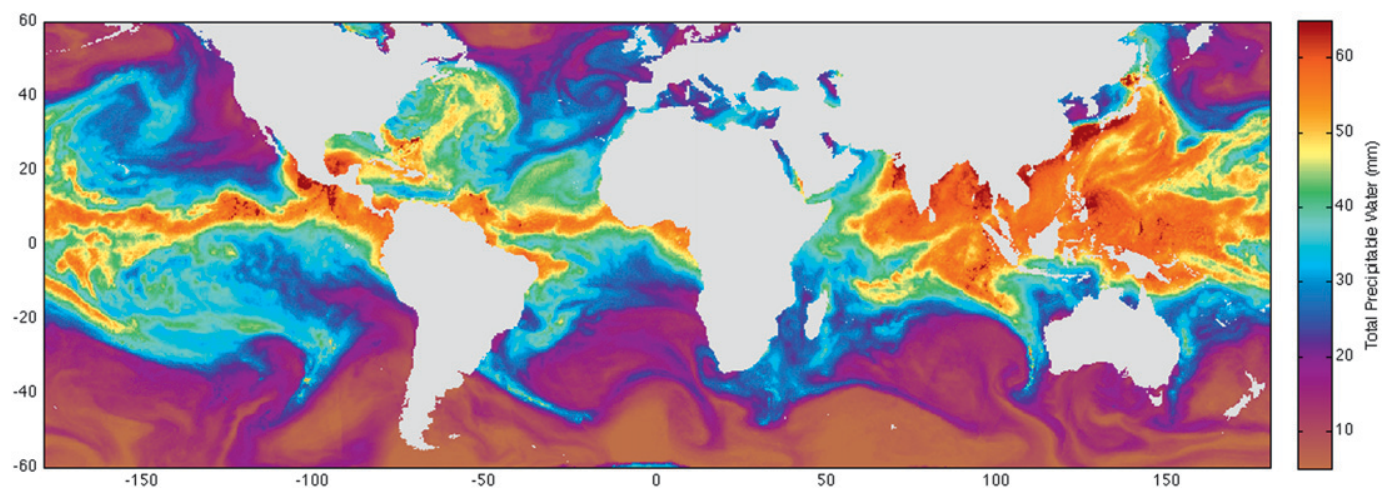

FIG. 3. Final product of the weighting process, valid at 1800 UTC 22 Jun 2009. 


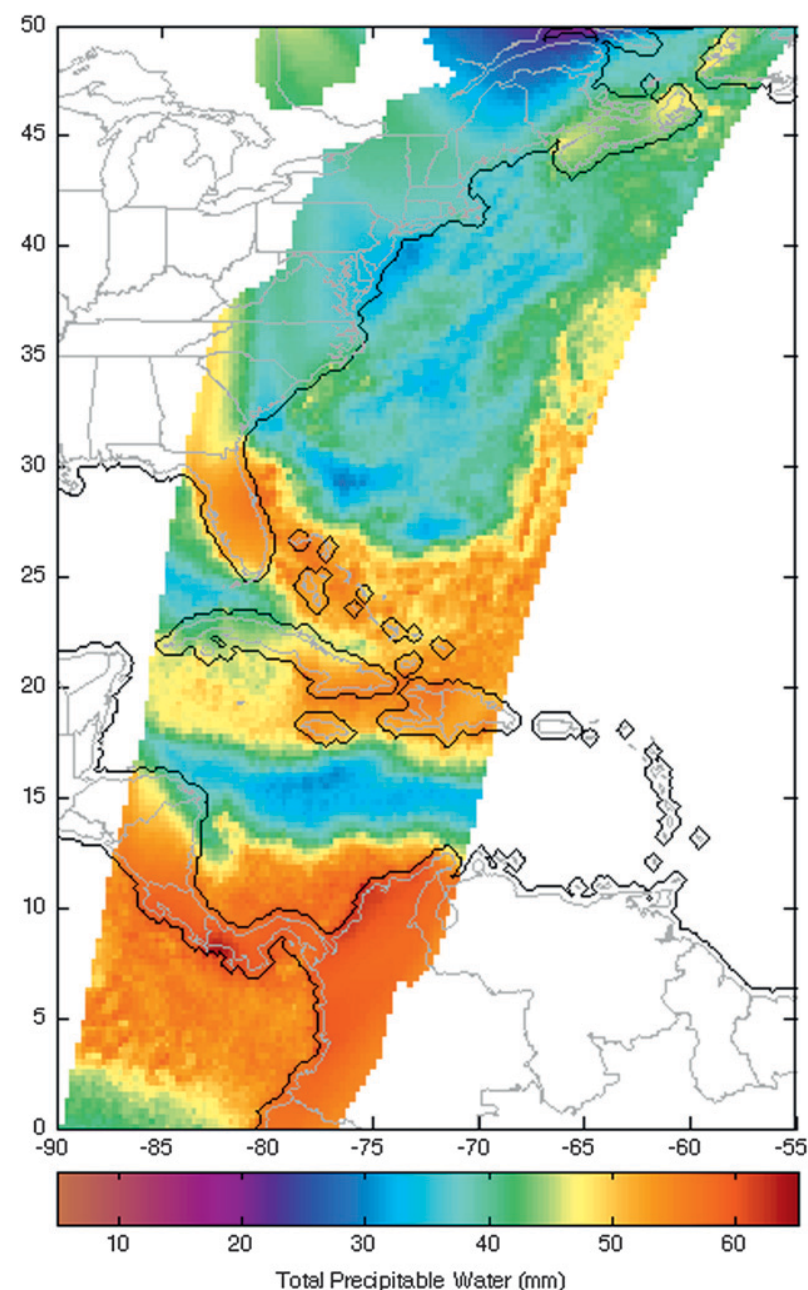

FIG. 4. Example of data extrapolation with inpainting. Extrapolated (nonretrieval) values are landward of the solid black contours.

interpolation [Eq. (1)]. Thus, in near-real-time applications, we use only the data nearest in time and earlier than the valid time for the product, which is equivalent

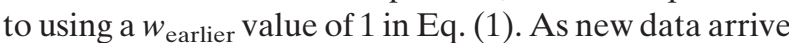
to populate the set of synthetic swaths at a given time, the later dataset is gradually introduced and updated into the product over the most recent $\sim 12 \mathrm{~h}$. In addition, only forecast wind fields are available for advection in the most recent hours, so every $12 \mathrm{~h}$ the real-time algorithm regenerates the advected data swaths using analysis wind fields when they become newly available. Updated final products are introduced only after an entire recomputation with new model fields, in order to prevent any operational artifacts.

In practice, this process first creates the real-time hourly product image as a mosaic of forward-advected swaths. Over the subsequent hours, the image at this time stamp is updated several times with more and more advected swaths, first with shorter forward-advection times, and then with a combination of forward and backward advection swaths. At the same time, newer real-time images are made with the forward-advected swaths of newer satellite data to complete a real-time animation of hourly images up to the present time.

\section{e. Current specifications}

The MIMIC-TPW product is derived at a temporal resolution of $1 \mathrm{~h}$ and at a spatial resolution of $0.25^{\circ}$. We have found that forward and backward swath advections can be realistic up to roughly $16 \mathrm{~h}$ out, so the MIMICTPW algorithm uses this as the maximum advection time for the set of synthetic advected swaths. The product is generated from a hierarchy of Matlab scripts running in a continuous loop to incorporate new satellite data and model wind fields in real time. These specifications-a product resolution of $0.25^{\circ} \times 0.25^{\circ}$ at 1 -h steps and a production of synthetic swaths up to $16 \mathrm{~h}$ away from the observation time-are within a comfortable margin of the computational limits of the devoted workstation to keep pace with real-time demonstrations. The current workstation is a Dell Precision T3400 with a $2.33-\mathrm{GHz}$ Intel Core 2 Duo E6550 processor.

In the subsequent sections, the unit of sample values is the $0.25^{\circ} \times 0.25^{\circ}$ grid cell. This allows direct matchups between advected swaths from different platforms. Although the grid cells are interpolated from the original data, it is at a similar resolution, which allows for the highest number of independent samples to use in the analysis.

\section{Product update frequency}

The performance of a composite product is heavily dependent on the data update frequency. We have found that the most straightforward way to measure this update frequency is through the relative distribution of the time gap between observations, sampled at each grid cell in the product. Figure 5 shows the best possible outcomes for a composite product created from combinations of 1,2,3, or 4 available satellites over the test period. As Fig. 5 shows, one satellite yields a distribution of time gaps mostly between 10 and $14 \mathrm{~h}$, due to the time between an overlap of the ascending and descending nodes of the satellite path. A small but significant peak in time gaps also appears at $24 \mathrm{~h}$, corresponding to the areas that update between successive ascending or descending passes. Many $(19.45 \%)$ of the grid cells do not have a time gap less than the $32 \mathrm{~h}$ necessary for a proper interpolation. These cells may still be filled with points extrapolated from nearby times; however, one of the endpoints (either earlier or later) will be missing because no data exist 

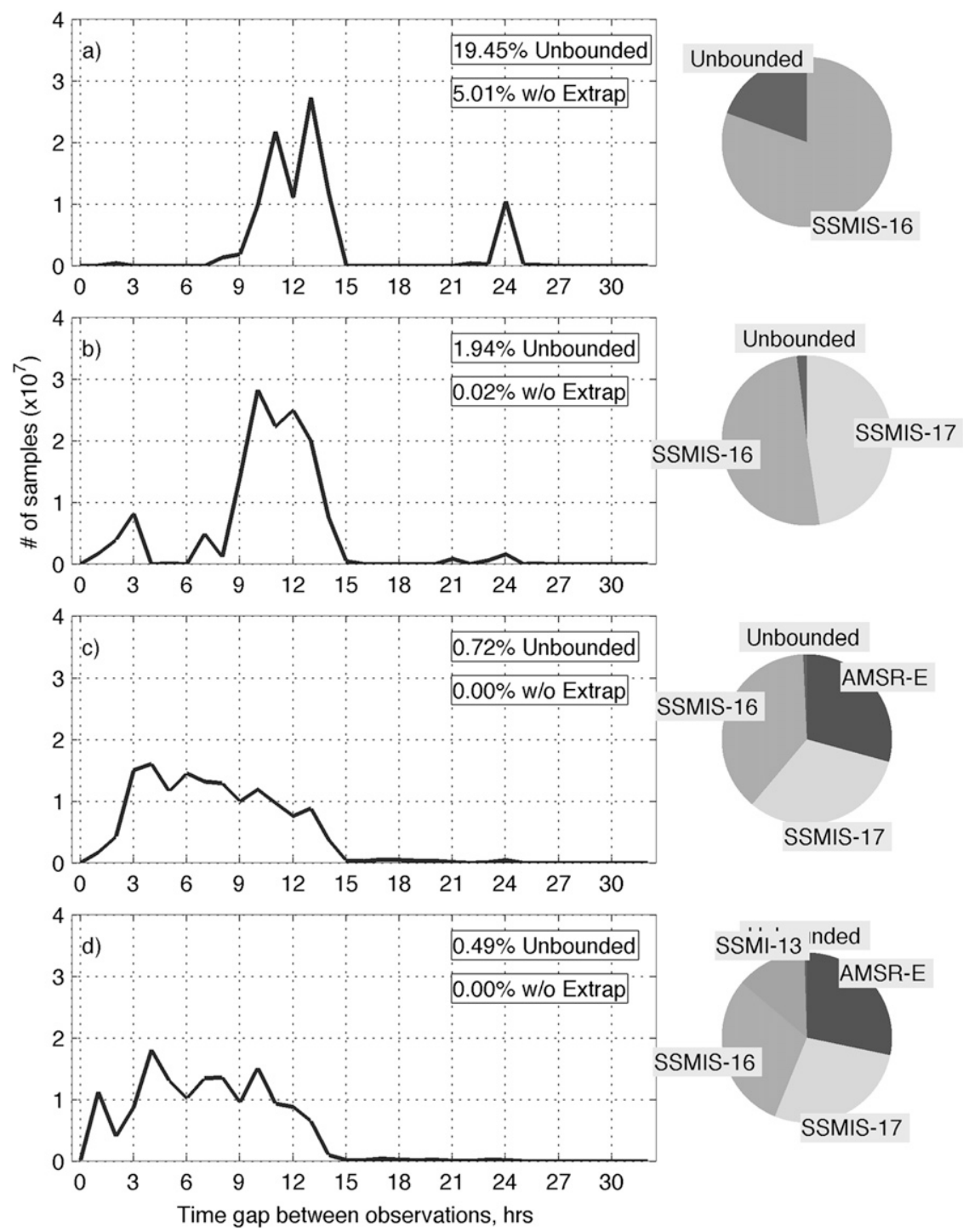

FIG. 5. Histograms of time gaps spanned by the MIMIC-TPW product gridcell samples during the 18-day test period for the best-case combinations of (a) 1, (b) 2, (c) 3, and (d) 4 satellites. Here, SSMI-13 indicates F-13 SSM/I, SSMIS-16/17 indicates F-16/-17 SSMI/S, AMSR-E indicates Aqua AMSR-E, Unbounded indicates points without both earlier and later endpoint values, and w/o Extrap indicates points without either earlier or later endpoint values.

within the $\pm 16 \mathrm{~h}$ operational limit of the final product. A smaller portion of grid cells $(5.01 \%)$ has no extrapolated data either (labeled as unbounded in Fig. 5) and appears as data gaps in the product. These regions of grid cells can be thought of as being "out of reach" of the synthetic advected swaths.

The best available combination of two satellites is the $F-16$ and $F-17 \mathrm{SSMI} / \mathrm{Ss}$, which have equator-crossing times $2 \mathrm{~h}$ apart. This allows the $\sim 1700-\mathrm{km}$-wide swaths to track one another roughly in tandem and cover almost the whole globe with time gaps of less than $15 \mathrm{~h}$. The wide peak between 9 and $14 \mathrm{~h}$ is due to the time gap between successive passes from either satellites' ascending or descending nodes. Only $1.94 \%$ of the grid cells cannot be interpolated between earlier and later swaths, and only $0.02 \%$ are lacking data altogether.

The time gap distributions of three- and four-satellite combinations are very similar because the fourth satellite, $F-13$, tracks so closely with the $F-16$ and $F-17$ that it is nearly redundant. In each of these cases, the Aqua AMSR-E breaks up the time gaps in the two-satellite 10-14-h peak to create a more even distribution of time gaps between 1 and $14 \mathrm{~h}$. The portion of grid cells without an interpolation is below $1 \%$, due mostly to artifacts at the 


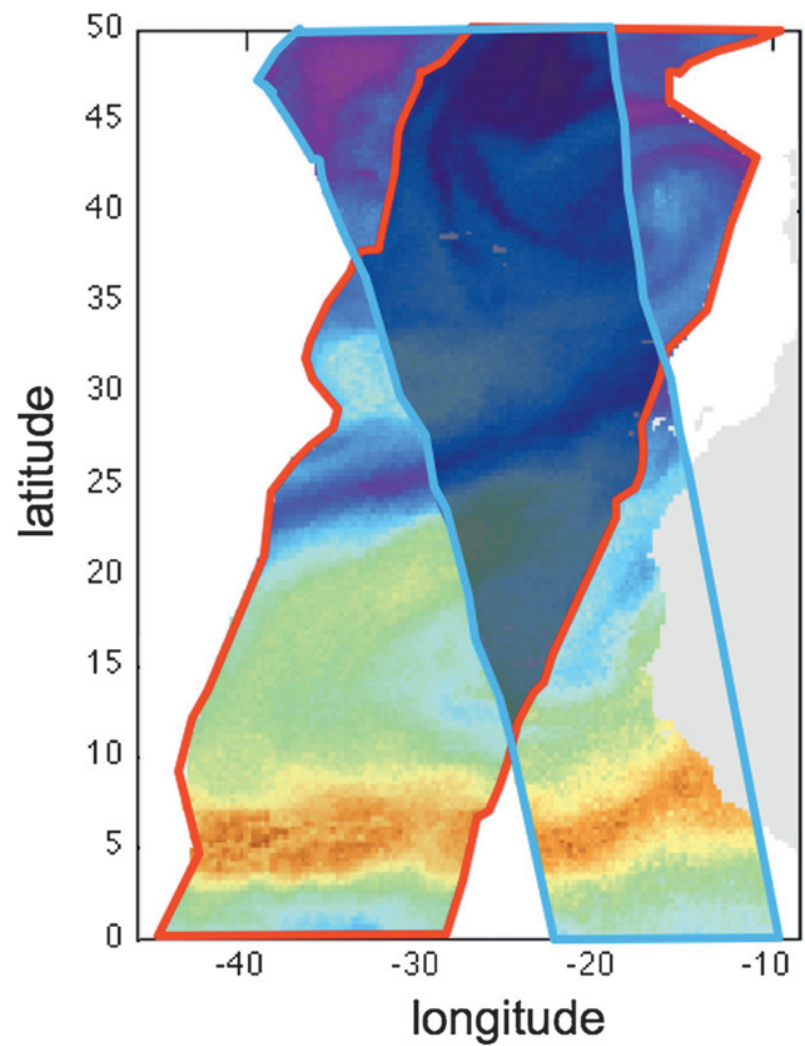

FIG. 6. Example of the components of the validation process The TPW swath outlined in red is the $F-16 \mathrm{SSMI} / \mathrm{S}$ swath from approximately 0922 UTC 22 Jun 2009, advected forward to the reference time of 1900 UTC 22 Jun 2009. The TPW swath outlined in blue is an observed $F-13 \mathrm{SSM} / \mathrm{I}$ retrieved field from within $30 \mathrm{~min}$ of the reference time. The tinted overlap region is a collection of grid cells tagged with an advection time of $+9.6 \mathrm{~h}$

coasts. (Regions near the coasts with seaward advection have no earlier data and spaces with landward advection have no later data; the motion of these artifacts would be particularly distracting without the data extrapolation technique described in section 2). Also, the portion of grid cells with no extrapolation data is effectively zero, although in operational mode there are sometimes periods of time when problems in the upstream data will cause gaps even in the historical product.

\section{Product validation}

\section{a. Method}

The redundancy in data coverage makes validation of the advection algorithm a very straightforward process. To measure the accuracy of an advected swath at a particular reference time, it simply needs to be compared to an overlapping portion of another swath whose observation time matches the reference time to within a margin of 30 min (Fig. 6). To minimize the contribution of the intercalibration error, we use only the SSM/I and SSMI/S satellites. This combination of satellites has frequent overlaps at time differences of less than $3 \mathrm{~h}$, and between 7 and $15 \mathrm{~h}$ (Fig. 7). The shortage of time differences between 4 and $6 \mathrm{~h}$ (inclusive) will need to be noted in the trends found in the validation, but it will be shown that this is not a significant problem in the analysis.

Data for validation are taken from hourly synthetic swaths of the MIMIC-TPW product from 2100 UTC 7 June 2009 to 0600 UTC 24 June 2009. This span of time generates nearly the maximum number of separate data points that can be managed in all of the analysis methods (4.9 million grid cells). A separate validation examines the accuracy of using forecast winds, and that validation covers a smaller set of data points (592 116 grid cells) because of a more limited availability of forecast winds in the archive ( $\sim 25 \%$ of the previous time period).

\section{b. Error trends}

Figure 8 shows that the mean average error (MAE) of all the advected swath data trends upward linearly with advection time. The shaded areas in Fig. 8 signify advection times that are rarely used in the end product, because a maximum time gap of $14 \mathrm{~h}$ indicates a maximum advection time of $7 \mathrm{~h}$ either before or after. Therefore, the most relevant range is between the shaded regions, where the trend in MAE varies between approximately 0.5 and $2 \mathrm{~mm}$. Note that the MAE does not approach 0 at an advection time of 0 because of minor residual errors that add to the overall error statistics, such as intercalibration, interpolation, and parallax.

A breakdown of MAE by zone (Fig. 9) reveals that the trends are similar in the northern midlatitudes, the tropics, and the southern midlatitudes. The error is slightly smaller in the southern midlatitudes because of the lower variance in TPW during the midlatitude winter. In addition, these trends show that the generally less accurate model winds in the Southern Hemisphere are not a serious impediment to algorithm performance.

A similar analysis of error was performed using only forecast winds to assess the relative increase in the error of the advected swaths when forecast winds are used in a realtime application setting (Fig. 10). Only forward advections are used in this comparison, because that is where forecast winds are predominantly applied in an operational application. Here, it is shown that product errors using analysis winds and forecast winds track very closely, and that the difference in MAE is only $0.13 \mathrm{~mm}$. Clearly, the shortterm forecast and analysis winds do not often diverge significantly enough over the oceans to make an impact in near-surface advections at $15 \mathrm{~h}$ or less.

Finally, an analysis of error by percentiles shows the magnitude of the relative outliers (Fig. 11). Again 

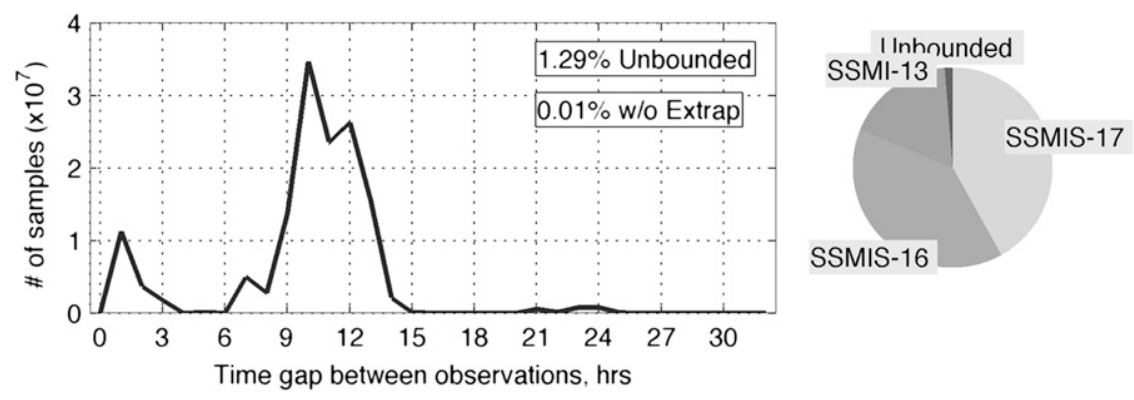

FIG. 7. Histogram of time gaps spanned by the MIMIC-TPW product grid cell samples during the 18-day validation period.

considering that the most common range of advection times is 0 to $7 \mathrm{~h}$, the error magnitude of even the 95th percentile is considered acceptable $(6 \mathrm{~mm}$ at $7 \mathrm{~h})$, because $6 \mathrm{~mm}$ is too small a change in color values on the product image to interfere with the user's interpretation. However, the errors at the 98th and 99th percentiles are significant near advection times of $7 \mathrm{~h}$. The most common source of this error is the extrapolation of TPW values near the coasts. A second source is intense convective events between the observation times of the advected swath and the validation swath. This is indeed a drawback of the algorithm, even though the saturation of TPW values in precipitating regions generally mitigates the differences between overlapping swaths. Convection can appear in the image animations as an artifact that is "pulsing" between observations rather than evolving continuously. However, when this artifact is understood, it is very unlikely to interfere in the user's interpretation of the end product. A final point worth noting is that unless the product is being computed in near-real time ( $\sim 9 \mathrm{~h}$ from the present), the error of the major outliers is usually subdued in the final product's weighted average of one extreme value with a second, more accurate value.

\section{Examples}

MIMIC-TPW can be used to study events from the local and hourly scales to the global and interannual scales. At the global scale, a sequence from 10 December 2009 to 7 April 2010 shows how the MIMIC-TPW (a)

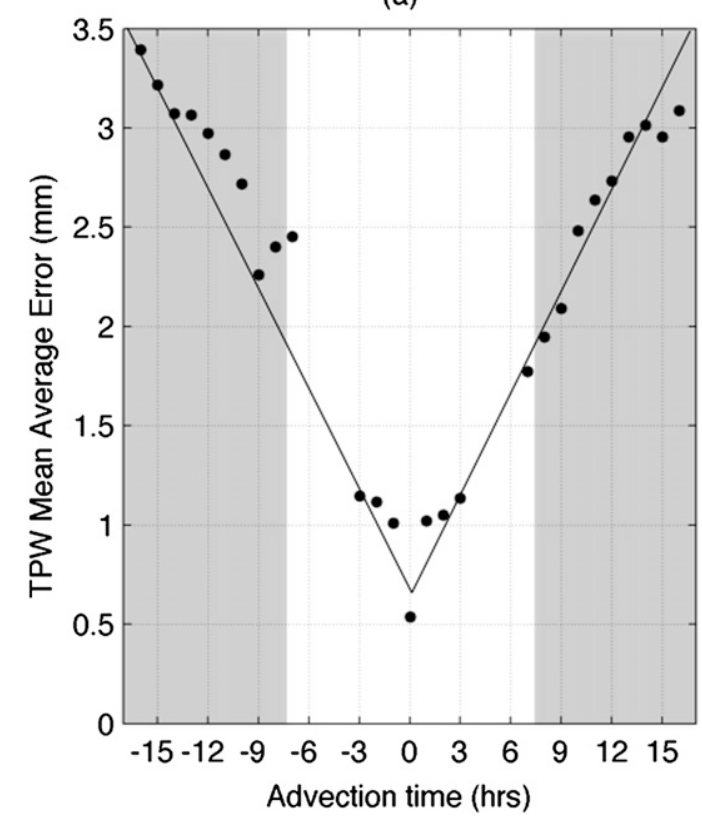

(b)

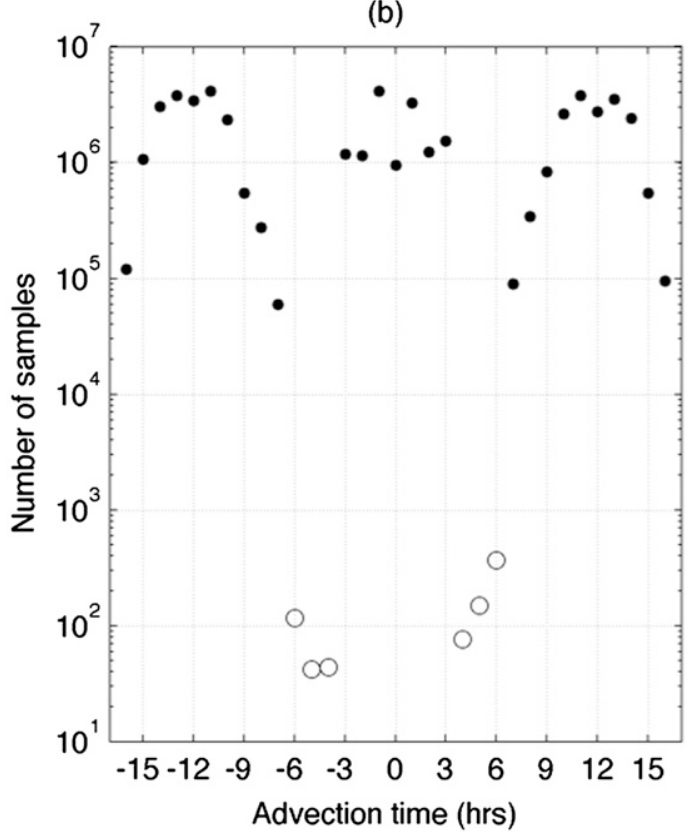

FIG. 8. Analysis of MIMIC-TPW error as a function of advection time: (a) MAE and (b) number of samples. Solid line is the trend in the data series, undersampled bins ( $<1000$ grid cell samples) are omitted from (a) and shown with open circles in (b), and the shaded area is discussed in the text. 
(a)

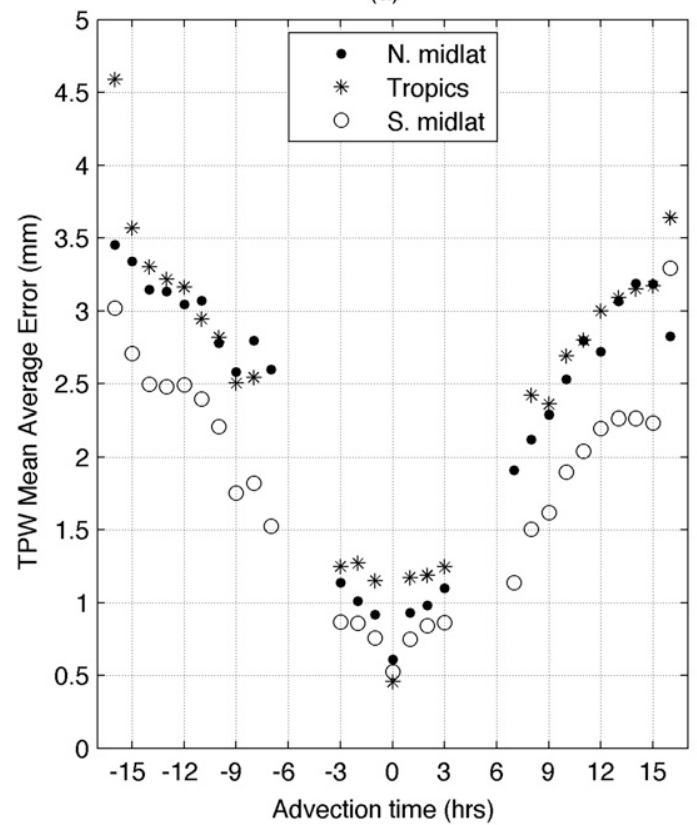

(b)

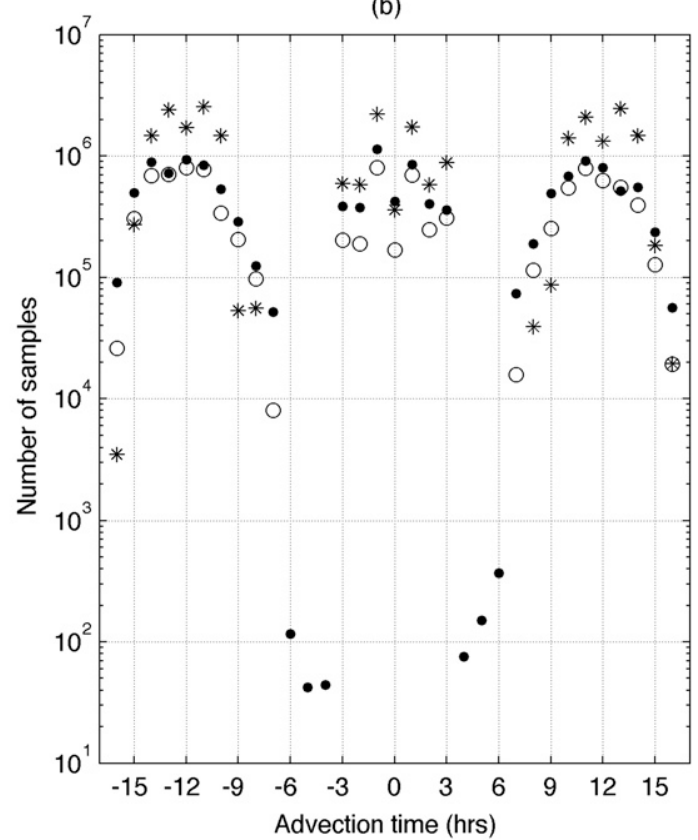

FIG. 9. The MIMIC-TPW error by zone: (a) MAE and (b) number of samples. Undersampled bins ( $<1000$ grid cell samples) are omitted from (a).

product reveals the patterns of behavior and evolution for the intertropical convergence zone (ITCZ), along with periods of tropical moisture pooling and transport into the midlatitudes (see animation 1 in the supplemental material at the Journals Online Web site: http://dx.doi.org/10.1175/2010JAMC2589.s1). Westwardmoving equatorial waves are readily apparent, and can be long lived. Also evident are a number of Southern Hemispheric tropical cyclones that were spawned from convergence zones in the South Pacific ITCZ over this period. Another salient pattern in this sequence is the powerful effect of subtropical anticyclones on the flow in the ITCZ. This is most apparent in the Atlantic, resulting in a high-amplitude zonal undulation.

All of the remaining examples are taken from 72-h sequences derived in a demonstration mode in real time for the ocean basins provided as regional products on the MIMIC-TPW Web site (http://cimss.ssec.wisc.edu/tropic/ real-time/tpw $2 /$ global $/$ main.html). If the reader is interested in other cases from July 2007 to the present, they are available in the archive section of the site in the same format as these examples.

An example from the North Atlantic basin (Fig. 12; see also animation 2 in the supplemental material at the Journals Online Web site: http://dx.doi.org/10.1175/ 2010JAMC2589.s2) shows the potential for using this product for tropical wave and cyclone forecasting. Three tropical waves are observed in the ITCZ beginning near Africa, the middle Atlantic, and South America and moving westward. In addition, the outline of a dry Saharan air layer (Dunion and Velden 2004) is suggested by TPW values below $30 \mathrm{~mm}$ and confirmed from other sources tracking westward from the western African coast. In addition, the edge of a polar air mass is clearly delineated between $40^{\circ}$ and $50^{\circ} \mathrm{N}$. This particular animation was cited multiple times by the National Hurricane Center in their Tropical Weather Discussion.

A tropical application example in the West Pacific basin (Fig. 13; see animation 3 in the supplemental

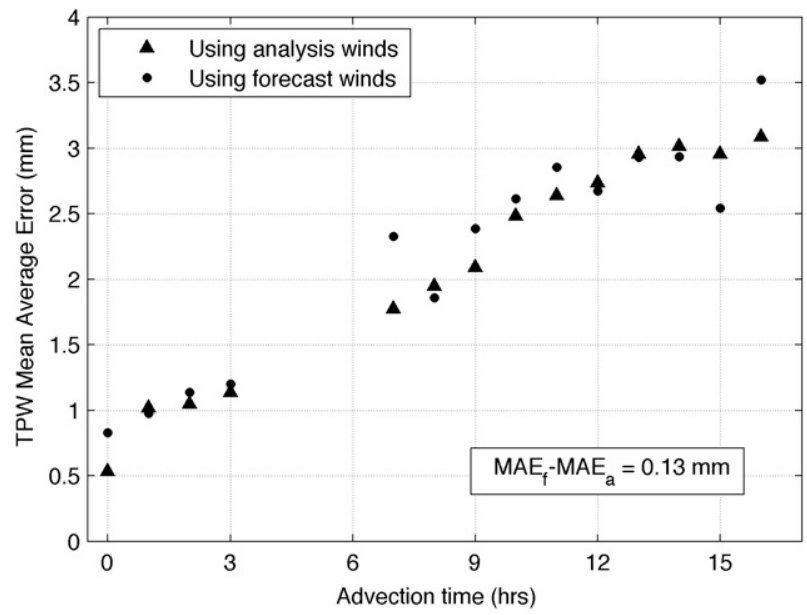

FIG. 10. Comparison of MIMIC-TPW error using analysis winds (triangle) and forecast winds (circle); undersampled bins $(<500$ grid cell samples) are omitted. 


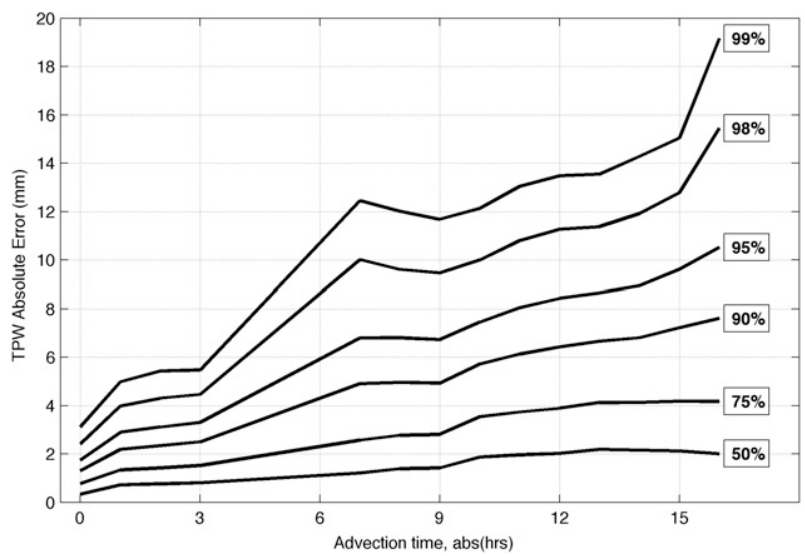

FIG. 11. The MIMIC-TPW error as a function of advection time (forward and backward) organized by percentile. (For example, the 99th percentile is the error magnitude larger than $99 \%$ of the points in the sample.)

material at the Journals Online Web site: http://dx.doi. org/10.1175/2010JAMC2589.s3) shows the progression of Typhoon Vamco $\left(\sim 25^{\circ} \mathrm{N}, \sim 155^{\circ} \mathrm{E}\right)$. This is significant because it shows the evolution of a tropical cyclone and the eventual part played by the polar front in extratropical transition. Here, it can be seen that the track of the typhoon quickly changes to an eastward direction around 1600 UTC 24 August, which is the same time that the dry barrier between the typhoon and the warm side of the front vanishes. As seen here, the ability of this product to present the continuous evolution of a TC without major artifacts demonstrates that the $1.25^{\circ}$ GFS-based wind field is sufficient even for driving the evolution of mesoscale structures given the algorithm's 1-14-h gap time.

Finally, a sequence from the Australia-New ZealandIndonesia region (Fig. 14; see animation 4 in the supplemental material at the Journals Online Web site: http://dx.doi.org/10.1175/2010JAMC2589.s4) shows a clear example of "atmospheric rivers" in the midlatitudes. The first event is associated with a small extratropical cyclone around $30^{\circ} \mathrm{S}, 160^{\circ} \mathrm{W}$ that subsequently weakens, but trails behind it an extensive plume of moisture that crosses the northern island of New Zealand from 0000 UTC 14 July through the end of the animation. (The train of moisture continued as a second extratropical cyclone intensified just east of New Zealand and finally moved away around 0000 UTC 18 July). A second plume of moisture impacts western Australia around 1200 UTC 15 July. The southern end of this plume is seen curling tightly around a low pressure center.

Two additional examples and description of atmospheric rivers impacting North America and the British Isles, respectively, have been documented by S. Bachmeier on the CIMSS satellite blog (http://cimss.ssec.wisc.edu/goes/ blog/archives/2489 and http://cimss.ssec.wisc.edu/goes/blog/ archives/3838).

\section{Conclusions}

This paper presents a method for displaying smooth, accurate, and high temporal resolution composites of polar satellite imagery of one specific quantity: total precipitable water. The resulting product increases the options for how polar-orbiting satellite-derived TPW can be used in weather analysis and near-real-time forecasting. This can be expanded into a broader message that many other, if not most other, polar satellite products can be likewise enhanced to great effect with blended advection. The simulations of section 4 showed that a full global product for any relatively conservative tracer is possible

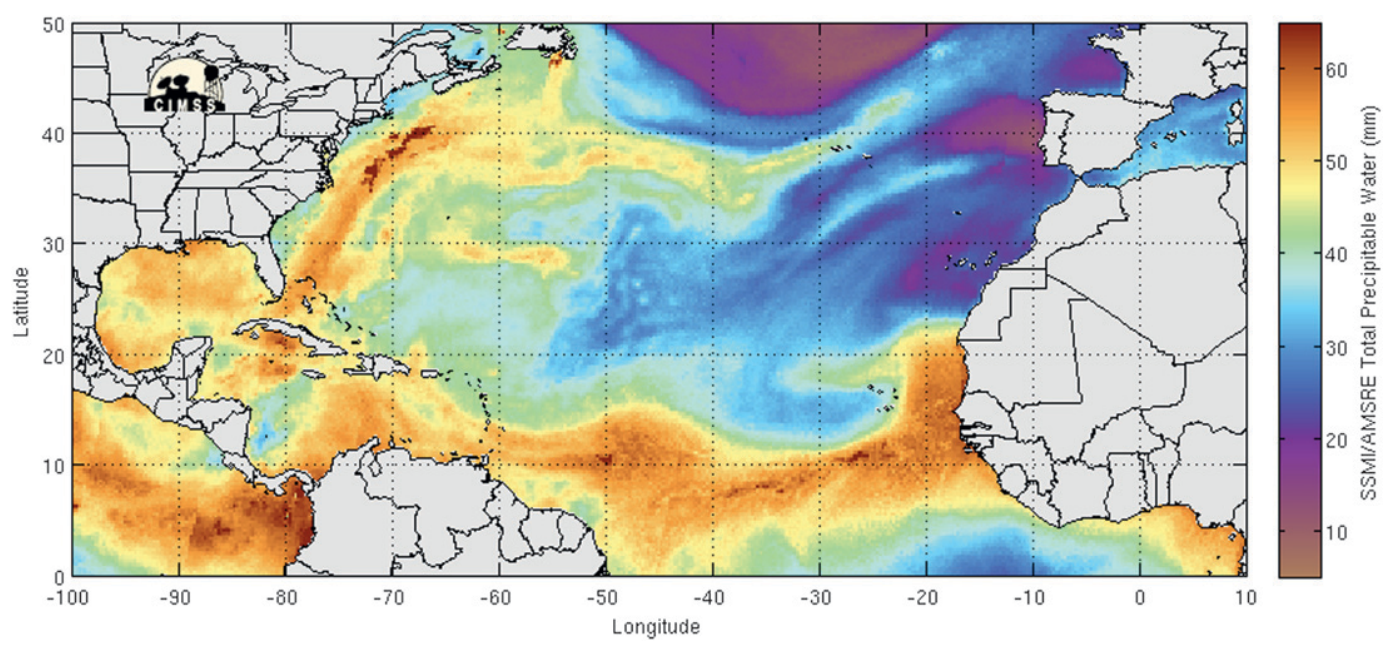

FIG. 12. The MIMIC-TPW product in the North Atlantic basin at 0700 UTC 28 Jul 2009. See also animation 2 (26-28 July) in the supplemental materials at the Journals Online Web site (http://dx.doi.org/10.1175/2010JAMC2589.s2). 


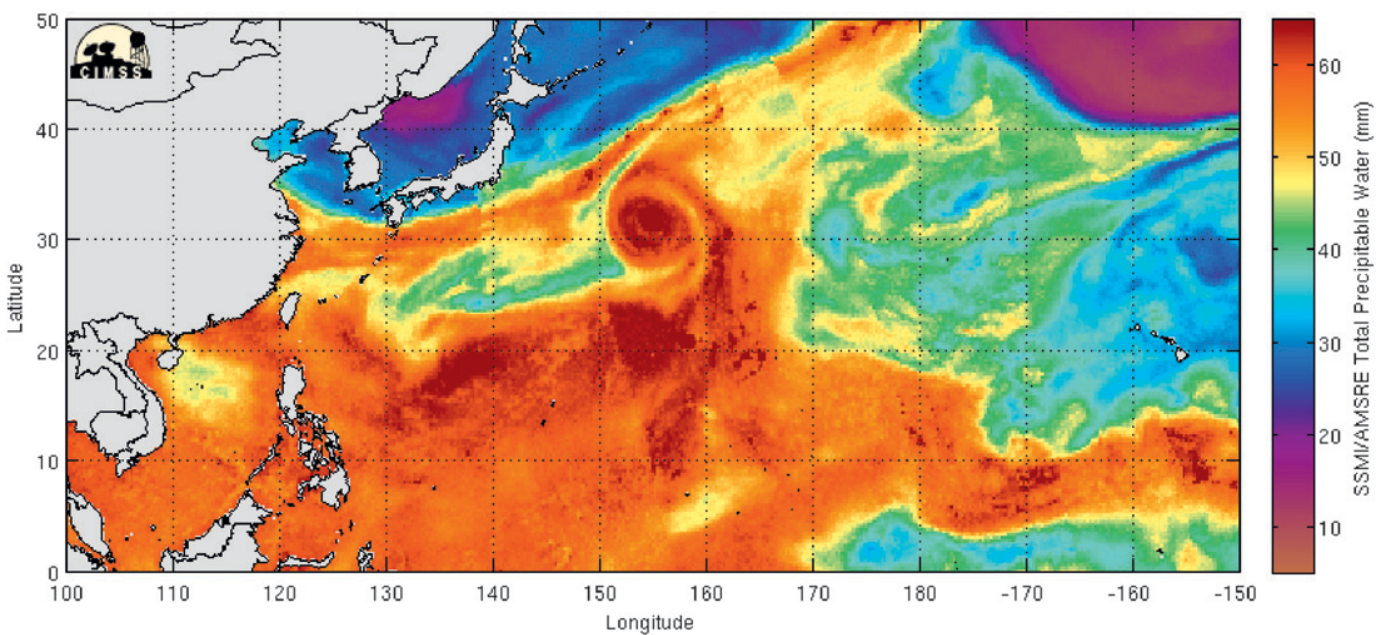

FIG. 13. The MIMIC-TPW product in the western Pacific basin at 0100 UTC 24 Aug 2009. See also animation 3 (22-24 August) in the supplemental materials at the Journals Online Web site (http://dx.doi.org/10.1175/2010JAMC2589.s3).

with only two satellites if the tracer lifetime does not exceed about $7 \mathrm{~h}$ (approximated with two 7-h extrapolations on either side of a 14-h gap). If the coverage increases with additional satellites, the maximum allowable tracer lifetime is further reduced, and the extent of this impact is dependent on the satellite orbit configuration.

There are several advantages of MIMIC-TPW over model-generated TPW, which extends into reasons why advective blending of satellite data can be preferable to modeled quantities in general. First, the advectively blended data are anchored directly to observations, and they can be interpreted as a broad collection of observations. Second, the user knows explicitly that the product is not oversmoothed. Third, the MIMIC-TPW product is routinely available at 1-h resolution globally and, in principle, can be customized to any temporal resolution required for a given application.

Other quantities where advective blending would be especially effective include microwave-based moisture and temperature retrievals, aerosols, and trace gases such as $\mathrm{NO}_{x}, \mathrm{O}_{3}, \mathrm{BrO}$, and $\mathrm{CO}_{2}$. In each case, a suitable vertically weighted wind profile would be needed for the corresponding advection function. Note that among these quantities mentioned, only the microwave-based products would be free of gaps and artifacts in the final product caused by cloud obstructions.

Similarly, advective blending could be used to create smoother animations of cloud motions around the poles

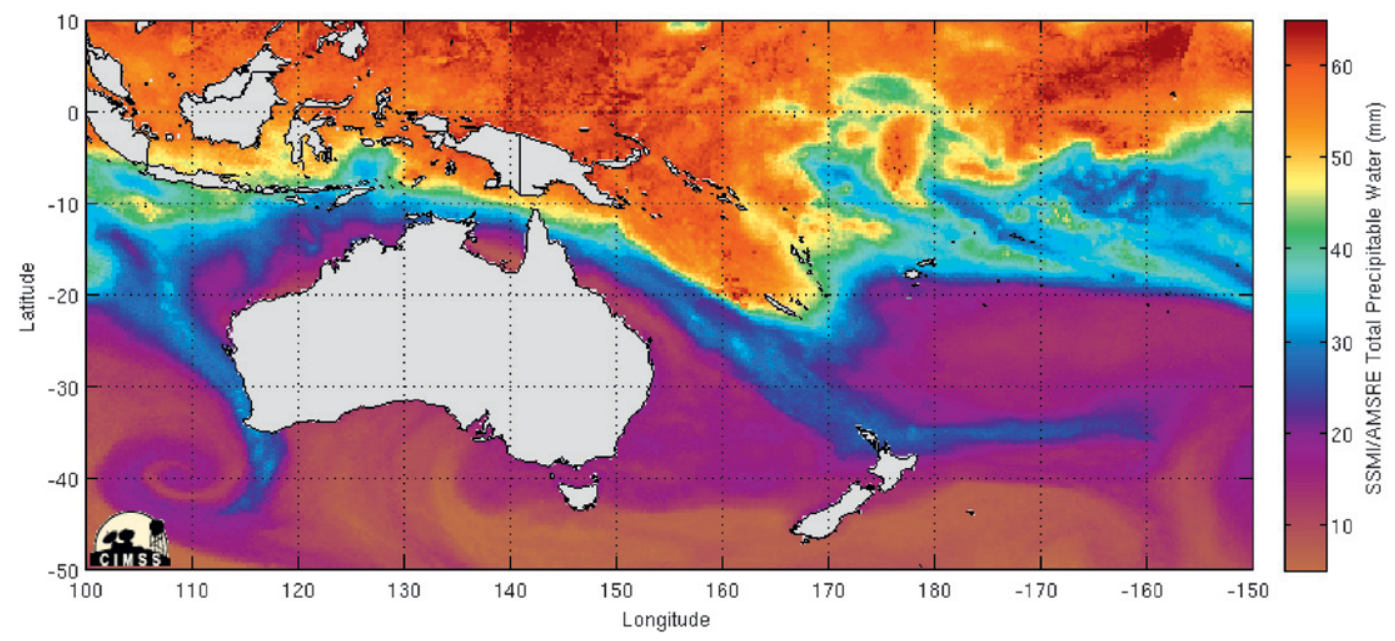

FIG. 14. The MIMIC-TPW product in the Australia-New Zealand-Indonesia basin at 1100 UTC 15 Jul 2009. See also animation 4 (13-15 July) in the supplemental materials at the Journals Online Web site (http://dx.doi.org/ 10.1175/2010JAMC2589.s4). 
(Lazzara et al. 2003). Finding a good advection function would be more challenging because of the large vertical distribution of clouds as well as the need to separate surface and cloud imagery. However, these problems would be partly alleviated by the large number of infrared and visible satellite-based sensors that can be used. With six or more satellites contributing to a product, no artifact would last more than a few hours.

The two major theoretical limitations of the advective blending method are the design of the advection function (already mentioned) and the effects of "sources" and "sinks" of the advected quantity. These could be either stationary or Lagrangian, because in either instance a source or sink will disrupt the assumed continuity of the quantity. The main source of TPW is the surface flux that occurs predominantly with intense convection. The examples in section 6 show that these fluxes can create noticeable artifacts in the product, but these are not severe enough to impede their interpretation. On the other hand, the sinks of TPW are more complicated to define, because although the reduction of water vapor occurs primarily through precipitation, the lower values of TPW in the global distribution are generally caused by subsidence and lower-tropospheric divergence. These processes are very slow with respect to the data refresh rate of MIMICTPW, so the sinks of TPW do not cause significant artifacts at all.

The MIMIC-TPW product continues to operate in a real-time demonstration mode at University of Wisconsin's CIMSS (http://cimss.ssec.wisc.edu/tropic/real-time/tpw2/ global/main.html) and receives regular updates to ensure reliability and maximize its impact on large-scale moisture analysis. Meanwhile, the satellite microwave data sources of TPW will continue to include the Defense Meteorological Satellite Program SSMI/S sensors and the NASA Aqua AMSR-E for as long as they remain activated.

Acknowledgments. This work was sponsored by the Oceanographer of the Navy through the PEO C41 PMW120 program office and administered through the Naval Research Laboratory (Jeff Hawkins). Access to real-time satellite data was made possible from the generous assistance of Paul Haggerty of the Science Technology Corporation (for AMSR-E) and Jeffery Tesmer of the Fleet Numerical Meteorology and Oceanography Center (for SSM/I, SSMI/S). Hal Woolf of CIMSS assisted in SSM/I decoding algorithms, and Jim Nelson provided support with data staging at CIMSS. We also thank three anonymous reviewers for their time and effort providing constructive comments on the manuscript's improvement.

\section{REFERENCES}

Alishouse, J. C., S. A. Snyder, J. Vongsathorn, and R. R. Ferraro, 1990: Determination of oceanic total precipitable water from the SSM/I. IEEE Trans. Geosci. Remote Sens., 28, 811-815.

Bertalmío, M., A. Bertozzi, and G. Sapiro, 2001: Navier-Stokes, fluid-dynamics, and image and video inpainting. Proc. Computer Vision and Pattern Recognition 2001, Hawaii, HI, IEEE, I-355.

Dunion, J. P., 2011: Rewriting the climatology of the tropical North Atlantic and Caribbean Sea atmosphere. J. Climate, in press.

- , and C. S. Velden, 2004: The impact of the Saharan air layer on Atlantic tropical cyclone activity. Bull. Amer. Meteor. Soc., 85, 353-365.

Germann, U., and I. Zawadzki, 2002: Scale dependence of the predictability of precipitation from continental radar images. Part I: Description of the methodology. Mon. Wea. Rev., 130, 2859-2873.

Grose, A., E. A. Smith, H.-S. Chung, M.-L. Ou, B.-J. Sohn, and F. J. Turk, 2002: Possibilities and limitations for quantitative precipitation forecasts using nowcasting methods with infrared geosynchronous satellite imagery. J. Appl. Meteor., 41, 763-785.

Hohti, H., J. Koistinen, P. Nurmi, E. Saltikoff, and K. Holmund, 2000: Precipitation nowcasting using radar-derived atmospheric motion vectors. Phys. Chem. Earth, 25B, 1323-1327.

Joyce, R. J., J. E. Janowiak, P. A. Arkin, and P. Xie, 2004: CMORPH: A method that produces global precipitation estimates from passive microwave and infrared data at high spatial and temporal resolution. J. Hydrometeor., 5, 487-503.

Kalnay, M. K., and W. E. Baker, 1990: Global numerical weather prediction at the National Meteorological Center. Bull. Amer. Meteor. Soc., 71, 1410-1428.

Kidder, S. Q., and A. S. Jones, 2007: A blended satellite total precipitable water product for operational forecasting. J. Atmos. Oceanic Technol., 24, 74-81.

Lazzara, M. A., C. R. Stearns, J. A. Staude, and S. L. Knuth, 2003: 10 years of Antarctic composite images. Preprints, Seventh Conf. on Polar Meteorology and Oceanography/Joint Symp. on High-Latitude Climate Variations, Hyannis, MA, Amer. Meteor. Soc., 9.4. [Available online at http://ams.confex.com/ ams/pdfpapers/60787.pdf.]

NASA MSFC, 2001: AMSR-E data management plan-August 2001. NASA Marshall Space Flight Center. [Available online at http://www.ghcc.msfc.nasa.gov/AMSR/data_management_plan. html.]

Newell, R. E., N. E. Newell, Y. Zhu, and C. Scott, 1992: Tropospheric rivers?-A pilot study Geophys. Res. Lett., 19, 2401-2404.

NOAA/OSDPD, 2002: General SSMIS information. NOAA/ Office of Satellite Data Processing and Distribution. [Available online at http://www.osdpd.noa.gov/PSB/IMAGES/ssmisdoc. htm.]

Piexoto, J. P., and A. H. Oort, 1992: Physics of Climate. SpringerVerlag, $520 \mathrm{pp}$.

Raytheon, 2000: SSM/I user's interpretation guide. Rep. UG32268900, 104 pp. [Available online at http://www.ncdc.noaa.gov/ oa/rsad/ssmi/fnoc-ssmi-manual.pdf.]

Wimmers, A. J., and C. S. Velden, 2007: MIMIC: A new approach to visualizing satellite microwave imagery of tropical cyclones. Bull. Amer. Meteor. Soc., 88, 1187-1196. 